\title{
Necrotising Fasciitis of the Lower Limb caused by Community-Acquired Methicillin-Resistant Staphylococcus aureus
}

\author{
CK Chan, MBBS, AM Merican, MS Orth, AM Nawar, MS Orth, YA Hanifah *, MSc, KL Thong**, Ph.D \\ Department of Orthopaedic Surgery, University Malaya Medical Centre, 50603 Kuala Lumpur, Malaysia \\ *Department of Medical Microbiology, University Malaya Medical Centre, 50603 Kuala Lumpur, Malaysia \\ **Institute of Biological Sciences, Faculty of Science, University of Malaya, 50603, Kuala Lumpur, Malaysia
}

\begin{abstract}
Necrotising fasciitis caused by Community-Acquired Methicillin-resistant Staphylococcus aureus (CA-MRSA) has emerged as a new entity. Although it is recognised worldwide, there have been no reported cases to date in Malaysia. We report a case of necrotising fasciitis of the left lower limb in an otherwise healthy 20 -year-old man. He presented with septic shock and despite the paucity of clinical signs in the limb, the infection was aggressive. Methicillin-Resistant Staphylococcus aureus (MRSA) was isolated from the deep fascia of the leg. Panton-Valentine leucocidin gene (PVL), which is a stable genetic marker for CA-MRSA strain, was positive in this case. This case of community acquired MRSA necrotising fasciitis is of concern and may herald the emergence of this resistant organism in Malaysia. Vigilant surveillance and microbiological monitoring is needed to follow this CAMRSA trend.
\end{abstract}

Key Words:

Necrotising Fasciitis, Community Acquired-MRSA,

CA-MRSA, Vancomycin

\section{INTRODUCTION}

Methicillin-resistant Staphylococcus aureus (MRSA) is typically thought to be a nosocomial pathogen and its incidence has increased markedly in the past few decades. Community-acquired Methicillin-resistant Staphylococcus aureus (CA-MRSA) has emerged as a new pathogen that usually causes skin and soft tissue infections in the community, and to a lesser extent, pneumonia, otitis media, bacteraemia and urinary tract infections ${ }^{1}$. There have been no reported cases of CA-MRSA cases or outbreaks in Malaysia to date. We present here a case in which we encountered a young and healthy patient who presented with left lower limb soft tissue infection with few clinical signs to suggest necrotising fasciitis. Microbiological results and the patient's history confirmed that the pathogen involved was CA-MRSA.

\section{CASE REPORT}

A 20-year-old man presented to the hospital with fever and a 5 -day history of pain in the left leg. The pain was initially localised to the left knee joint and was associated with redness of the skin. The patient denied any recent injury to his left knee and reported no previous history of respiratory or genitourinary infections nor previous hospitalisations. He consulted a general practitioner and also sought alternative medicine treatment, however his condition did not improve. He developed fever with chills and rigour on day four, and when his fever and leg pain did not improve on day five, he sought medical treatment.

At presentation, the patient looked lethargic and was severely dehydrated. A fever of $38^{\circ} \mathrm{C}$ was accompanied by hypotension $(80 / 40 \mathrm{mmHg})$ and tachycardia $(140-150 \mathrm{bpm})$. Mild erythoema areas was present on the medial aspect of the left knee measuring approximately $10 \mathrm{~cm} \times 3 \mathrm{~cm}$. Tenderness was maximal at the posterior aspect of the knee and mild tenderness was elicited at the posterior aspect of the thigh and circumferentially in the leg. The left knee was held flexed at $30^{\circ}$ and the patient could only flex his knee up to $110^{\circ}$.

Radiographs of the left lower limb were normal. Ultrasound of the left lower limb revealed significant thickening of the subcutaneous tissues over the anterior and posterior aspects of the distal left thigh, knee and upper leg suggestive of cellulitis of the lower limb. A CT scan showed thickening of the leg fascia extending to the lateral thigh but did not reveal any other abnormalities. The patient's white cell count was $7.8 \times 10^{9} / \mathrm{L}$, erythrocyte sedimentation rate $(\mathrm{ESR})$ was 81 $\mathrm{mm} / \mathrm{hr}$, and C-reactive protein (CRP) was $14.0 \mathrm{mg} / \mathrm{dL}$.

Despite adequate rehydration, he remained hypotensive. Triple inotropes (dopamine, dobutamine and noradrenalin) were initiated and he was admitted to the Intensive Care Unit (ICU). Intravenous cloxacillin 1 gram qid and C-Penicillin 2.4 mega-units qid were initially administered, but due to signs of severe sepsis this was later changed empirically to Tazocin $7.5 \mathrm{mg}$ tid (piperacillin/ tazobactam). 


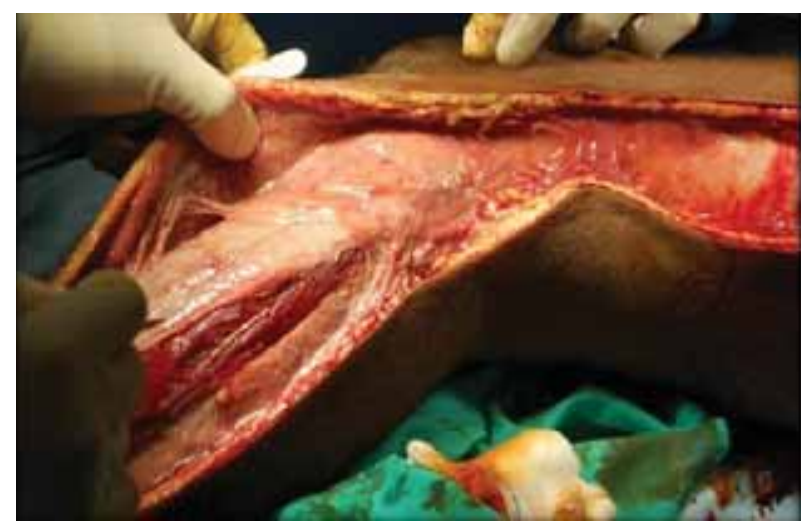

Fig. 1: There were extensive areas of necrosis and suppuration of the leg fascia circumferentially extending into the popliteal space up to the lateral thigh. The deep muscles of the lower limb and the periosteum overlying the subcutaneous surface of the tibia were healthy.

On the following day, the patient's clinical condition continued to deteriorate. He required increasing doses of inotropes to maintain his blood pressure and continuous positive airway pressure (CPAP) to maintain his oxygenation. Arterial blood gas results showed deterioration to metabolic acidosis. The erythoematous area of the limb diminished and the mild tenderness was now reduced to the popliteal area only. Despite improvement of the skin appearance, there was loss of sensation to pin prick circumferentially in the leg. Sensation in the foot remained intact. His continued deterioration and the loss of sensation were consistent with a diagnosis of necrotising fasciitis and emergency surgery was performed without further delay. The surgical findings are shown in figure 1.

Microbiological cultures of the fascia from the limb grew Methicillin-resistant Staphylococcus aureus (MRSA). Cultures were resistant to methicillin, erythromycin and ciprofloxacin, and sensitive to trimethoprimsulphamethoxazole, rifampicin, fucidic acid, gentamicin, vancomycin and clindamycin. Vancomycin had been started intraoperatively empirically based on the surgeon's suspicion of a staphylococcal infection and this was continued. The MRSA was found to have the Panton-Valentine Leucocidin (PVL) and mec A genes. However, the organism was nontypable for staphylococcal cassette chromosome mec (SCCmec: I $-\mathrm{V}$ ). Over the next four days, the patient underwent three more debridements for removal of more necrotic tissue. On day nine, the infection was controlled and no further necrosis was observed. The patient made a full recovery and was discharged from the hospital on day thirtynine of hospitalisation. Vancomycin was administered for total of five weeks, followed by oral fusidic acid and rifampicin for another two weeks. Split skin grafting was performed later and the wounds healed uneventfully.

\section{DISCUSSION}

Wound cultures revealed mono-microbial MRSA infection in this patient. This infection was due to communityacquired according to criteria described by the Centre for Disease Control and Prevention (CDC). According to the CDC, CA-MRSA is MRSA that had been isolated from patients who have no history of 1) positive culture for MRSA from any site obtained more than 48 hours after admission to a hospital (if hospitalised); 2) prior MRSA infection or colonization; 3) hospitalisation, surgery, residency in a longterm care facility, haemodialysis, or peritoneal dialysis within the past year; or 4) current indwelling percutaneous devices or catheters ${ }^{2}$.

Community-acquired methicillin-resistant Staphylococcus aureus (CA-MRSA) strains have demonstrated genetic and geographical diversity. It has been reported that there are genetic differences between CA-MRSA in Asian countries ${ }^{3}$. Most strains isolated in Southeast Asia Countries such as Singapore, Indonesia and Thailand were SCCmec type III or type IIIA. However, the MRSA isolated in this case was nontypable for any SCCmec. Panton-Valentine Leucocidin (PVL) is a pore-forming cytotoxin that targets human mononuclear and polymorphonuclear cells. It is reported that PVL is a stable genetic marker in CA-MRSA ${ }^{4}$. In this case, the isolated MRSA was PVL positive which is rare in Malaysia 5. This MRSA carried the mecA gene as well, which codes for methicillin-resistance.

Most of the MRSA cases isolated in Southeast Asia have been multiple-antibiotic resistant. Similarly, the isolated MRSA in this case was resistant to methicillin, erythromycin and ciprofloxacin and sensitive to trimethoprimsulphamethoxazole, rifampicin, fucidic acid, gentamicin, clindamycin and vancomycin with intermediate effect from piperacilin/ tazobactam. This case of CA-MRSA is a cause for concern as its emergence poses potential problems in clinical practice. Conventional use of cephalosporins may fail in the treatment of community-acquired skin and soft tissue infections. As with all MRSAs, there is a limited choice of antibiotics that can be used successfully for treatment. Vancomycin is still the antibiotic of choice for serious infections and the development of resistance with the emergence of vancomycin-resistant Staphylococcus aureus (VRSA) is of major concern worldwide. In view of the emergence of this new strain, CA-MRSA could replace outbreaks of methicillin-sensitive Staphylococcus aureus in the community setting. We suggest that close surveillance and awareness of this strain in addition to good infection control procedures are essential to monitor the spread of antimicrobial resistance profiles, and association with invasive soft tissue infection. 


\section{REFERENCES}

1. Naimi TS, LeDell KH, Como-Sabetti K, Borchardt SM, Boxrud DJ et al. Comparison of Community-and Health Care-associated Methicillin-Resistant Staphylococcus aureus infection. JAMA 2003; 290: 2976-84.

2. Community-Associated Methicillin-Resistant Staphylococcus aureus in Minnesota, Disease Control Newsletter, Minnesota Department of Health. 2004; 32: 61-72.

3. Ko KS, Lee JY, Suh JY, Oh WS, Peck KR, Lee NY et al. Distribution of Major Genotypes among Methicillin-Resistant Staphylococcus aureus Clones in Asian Countries. J Clin Microbiol 2005; 43: 421-6.

4. Vandenesch, F, Naimi T, Enright MC, Lina G, Nimmo GR, Heffernan H et al. Community-acquired methicillin-resistant Staphylococcus aureus carrying Panton-Valentine leukocidin genes: worldwide emergence. Emerg Infect. 2003; 9: 978-84.

5. Thong KL, Junnie J, Liew FY, Yusof MY, Hanifah YA. Antibiograms and Molecular Subtypes of Methicillin-Resistant Staphylococcus aureus in Local Teaching Hospital, Malaysia. J Microbiol Biotechnol 2009; 19: 1265-70. 